$$
\text { "bacso" — 2006/6/22 — 18:13 — page } 167 \text { — \#1 }
$$

\title{
Notes on the representational possibilities of projective quadrics in four dimensions
}

\author{
SÁndor BÁcsó and Zoltán SZILASI
}

\begin{abstract}
The paper deals with hyper-quadrics in the real projective 4-space. According to [1] there exist 11 types of hypersurfaces of $2^{\text {nd }}$ order, which can be represented by 'projective normal forms' with respect to a polar simplex as coordinate frame. By interpreting this frame as a Cartesian frame in the (projectively extended) Euclidean 4-space one will receive sort of Euclidean standard types of hyper-quadrics resp., hypersurfaces of $2^{\text {nd }}$ order: the sphere as representative of hyper-ellipsoids, equilateral hyper-hyperboloids, and hyper-cones of revolution. It seems to be worthwhile to visualize the "typical" projective hyper-quadrics by means of descriptive geometry in the (projectively extended) Euclidean 4-space using Maurin's method [4] or the classical (skew) axonometric mapping of that 4-space into an image plane.
\end{abstract}

Key words and phrases: projective quadrics, Euclidean and projective spaces, Maurin's projection, axonometry.

ZDM Subject Classification: G00, G70, G80.

\section{Introduction}

In the professional training in descriptive geometry the treatment of conics and quadrics in the 2 and 3 dimensional real projective space is of central importance. The classification of projective quadrics (based on the rank and signature as projective invariants) can be carried out similarly in higher dimensions as well [1]. A quadric in the four-dimensional real projective space is the set of points Supported by National Science Research Fundation OTKA No. T 48878.

Copyright (C) 2006 by University of Debrecen 
whose homogeneous coordinates $\left[x_{1}, x_{2}, x_{3}, x_{4}, x_{5}\right]$ satisfy the equation

$$
\sum_{I, K=1}^{5} a_{I K} x_{I} x_{K}=0
$$

where $\left(a_{I K}\right)$ is a $5 \times 5$ symmetric real matrix.

According to the rank and the signature of the quadratic form $Q(x)=$ $\sum_{I, K=1}^{5} a_{I K} x_{I} x_{K}$, we have the following types of projective quadrics in the fourdimensional real projective space:

\begin{tabular}{|r|c|l|l|l|}
\hline & rank & signature & projective equation & Euclidean equation \\
\hline 1. & $R=5$ & $S=5$ & $x_{1}^{2}+x_{2}^{2}+x_{3}^{2}+x_{4}^{2}+x_{5}^{2}=0$ & $x^{2}+y^{2}+z^{2}+t^{2}=-1$ \\
\hline 2. & $R=5$ & $S=3$ & $x_{1}^{2}+x_{2}^{2}+x_{3}^{2}+x_{4}^{2}-x_{5}^{2}=0$ & $x^{2}+y^{2}+z^{2}+t^{2}=1$ \\
\hline 3. & $R=5$ & $S=1$ & $x_{1}^{2}+x_{2}^{2}+x_{3}^{2}-x_{4}^{2}-x_{5}^{2}=0$ & $x^{2}+y^{2}+z^{2}-t^{2}=1$ \\
\hline 4. & $R=4$ & $S=4$ & $x_{1}^{2}+x_{2}^{2}+x_{3}^{2}+x_{4}^{2}=0$ & $x^{2}+y^{2}+z^{2}+t^{2}=0$ \\
\hline 5. & $R=4$ & $S=2$ & $x_{1}^{2}+x_{2}^{2}+x_{3}^{2}-x_{4}^{2}=0$ & $x^{2}+y^{2}+z^{2}-t^{2}=0$ \\
\hline 6. & $R=4$ & $S=0$ & $x_{1}^{2}+x_{2}^{2}-x_{3}^{2}-x_{4}^{2}=0$ & $x^{2}+y^{2}-z^{2}-t^{2}=0$ \\
\hline 7. & $R=3$ & $S=3$ & $x_{1}^{2}+x_{2}^{2}+x_{3}^{2}=0$ & $x^{2}+y^{2}+z^{2}=0$ \\
\hline 8. & $R=3$ & $S=1$ & $x_{1}^{2}+x_{2}^{2}-x_{3}^{2}=0$ & $x^{2}+y^{2}-z^{2}=0$ \\
\hline 9. & $R=2$ & $S=2$ & $x_{1}^{2}+x_{2}^{2}=0$ & $x^{2}+y^{2}=0$ \\
\hline 10. & $R=2$ & $S=1$ & $x_{1}^{2}-x_{2}^{2}=0$ & $x^{2}-y^{2}=0$ \\
\hline 11. & $R=1$ & $S=1$ & $x_{1}^{2}=0$ & $x^{2}=0$ \\
\hline
\end{tabular}

Column 4 shows the inhomogeneous equations of the standard hypersurfaces of $2^{\text {nd }}$ order with respect to a coordinate frame using the hyperplane $x_{5}=0$ as the ideal hyperplane at infinity. That will say that, in the regular cases $1,2,3$, the hyper-quadrics have a "centre" positioned at the origin of the frame; paraboloid type hyper-quadrics are not listed up. Column 4 contains cones with the origin as the vertex (cases $4,5,6$ ), but no cylinders. The cases 7,8 describe cones with a one-dimensional vertex coinciding with the $t$-axis; the cases 9 and 10 mean a pair of conjugate complex resp. real hyperplanes as 'cones' with the $z t$-plane as 2-dimensional vertex. Finally, case 11 means a single hyperplane, which has to be counted twice as a hyper-surface with singular points only.

Note that column 4 does not give a complete affine or Euclidean classification of hyper-quadrics! 


$$
\text { "bacso" — 2006/6/22 — 18:13 — page } 169 \text { — \#3 }
$$

From the viewpoint of classical descriptive geometry we have to distinct real objects from those which are essentially imaginary. Thus only the classes $2,3,5$, 6,8 have real branches. Of course the set of singular points of the hypersurfaces always is a real linear subspace. A "real" visualization of an imaginary hyperquadric $\Phi$ usually is based on the product of the (real) elliptic polarity to $\Phi$ and the reflection at the centre of $\Phi$, which is a hyperbolic polarity such that the set of self conjugate points is a real hyper-quadric $\Phi^{r}$. It is called the "real substitute" of $\Phi$. Taking this into consideration it is sufficient to treat the visualization problem for regular hyper-quadrics only in the cases 2 and 3 .

In this paper we will use Maurin's generalization of Monge's method [4] as well as (skew) axonometry to visualize a hyper-quadric $\Phi$ of type 2 (hyper-sphere) and 3 (equilateral hyper-hyperboloid).

In our opinion the students of mathematics and informatics faculties take an interest in the computer aided representation of geometric figures. At the same time, the fourth and higher dimensions are still quite mysterious for an average student. We hope our paper will demonstrate that there is no mystery; figures in four (and higher) dimensions can also be efficiently represented by the tools of descriptive geometry. An excursion into four dimensions has another advantage: it promotes a better understanding of our three-dimensional universe.

\section{Representation of the 4-dimensional sphere in 2-space by Maurin's method}

Monge's method of representing the Euclidean 3-spce in two dimensions was extended to the Euclidean 4-space by J. Maurin [4]; see also [5, 6, 7, 8]. A very clear account of Maurin's method is available in L. Gyarmathi's paper [2]. To make our paper as self-contained as possible, we also present a brief description of Maurin's projection. The starting configuration is a perpendicular coordinate tetrahedron $(0 x y z t)$ in the 4-dimensional Euclidean space. The role of planes of projections is played by the three coordinate planes $[x y],[x z],[x t]$. A point of the 4-dimensional Euclidean space is represented as follows:

(1) We construct the orthogonal projections $P^{\prime}, P^{\prime \prime}, P^{\prime \prime \prime}$ of $P$ onto $[x y],[x z]$ and $[x t]$ respectively.

(2) We rotate around the axes two of the image planes into the remaining one.

Then the three projections $P^{\prime}, P^{\prime \prime}$ and $P^{\prime \prime \prime}$ of $P$ will be located on a straight line perpendicular to the $x$-axis. Thus a point $P(x, y, z, t)$ is represented by the triplet 
$P^{\prime}(x, y), P^{\prime \prime}(x, z)$ and $P^{\prime \prime \prime}(x, t)$. The three planes of projection will be denoted by $K_{1}, K_{2}$ and $K_{3}$ in the following.

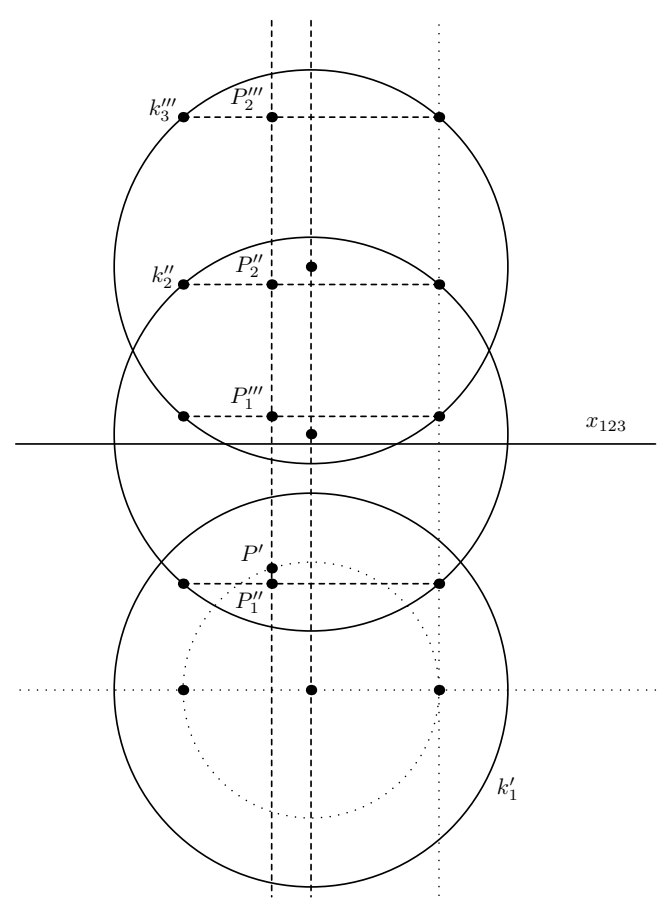

Figure 1

Now we turn to the representation of the three-dimensional real sphere belonging to the class 2 of projective quadrics given by the equation

$$
x^{2}+y^{2}+z^{2}+t^{2}=1
$$

by Maurin's method. The intersection of the sphere and the $[x y]$-plane is the circle given by the equation

$$
x^{2}+y^{2}=1 \quad(z=0, t=0) .
$$

The first projections of the other points of the sphere are interior points of this circle. Indeed, if $z^{2}+t^{2}>0$, then the relation

$$
x^{2}+y^{2}=1-z^{2}-t^{2}
$$




$$
\text { "bacso" — 2006/6/22 — 18:13 — page } 171 \text { — \#5 }
$$

implies that $x^{2}+y^{2}<1$. Similarly, the second and third projections of the points of the sphere also constitute discs of radius 1 .

It is clear from the construction that the three projections of a sphere whose center differs of the origin are also three circles $k_{1}^{\prime}, k_{2}^{\prime \prime}, k_{3}^{\prime \prime \prime}$ of the same radius with centers on the same ordering line. Given a point $P^{\prime}$ in the interior of circle $k_{1}^{\prime}$ one can determine those points of the sphere whose first projection is the point $P^{\prime}$. The problem can be reduced to the three dimensional case since projecting the sphere to the three-dimensional hyperplane $\left[K_{1}, K_{2}\right]$ spanned by the first and the second planes of projection we obtain a two-dimensional sphere. In this sphere, by the well-known method of parallel circles, one can construct two points $P_{1}^{\prime \prime}$, $P_{2}^{\prime \prime}$ such that their first projection is the given point $P^{\prime}$. In the next step we project our four-dimensional sphere to the hyperplane $\left[K_{1}, K_{3}\right]$, and repeat the preceding process; this leads to the points $P_{1}^{\prime \prime \prime}, P_{2}^{\prime \prime \prime}$. The triples

$$
\left(P^{\prime}, P_{1}^{\prime \prime}, P_{1}^{\prime \prime \prime}\right),\left(P^{\prime}, P_{1}^{\prime \prime}, P_{2}^{\prime \prime \prime}\right),\left(P^{\prime}, P_{2}^{\prime \prime}, P_{1}^{\prime \prime \prime}\right),\left(P^{\prime}, P_{2}^{\prime \prime}, P_{2}^{\prime \prime \prime}\right)
$$

so obtained represent the points of the three-dimensional sphere.

The parallel circles applied in the process have a clear geometric meaning also in four-space, since the three-dimensional sphere, as a hypersurface of revolution in $\mathbb{R}^{4}$, is built up of two-dimensional 'parallel spheres'.

\section{Representation of projective quadrics of class 3 by Maurin's method}

We can obtain a more clear picture also of the other classes of hyperquadrics in $\mathbb{R}^{4}$ determining their intersections with the hyperplanes spanned by pairs of planes of projections. In this section we apply a version of the above technique to the third class of projective quadrics. By this method we can have a more vivid picture on the other classes as well.

The inhomogeneous equation of a quadric belonging to the class 3 is

$$
x^{2}+y^{2}+z^{2}-t^{2}=1 .
$$

Intersecting with the hyperplane

$$
\left[K_{1}, K_{3}\right]: z=0
$$

we get a hyperboloid of one sheet with equation

$$
x^{2}+y^{2}-t^{2}=1 \text {. }
$$




$$
\text { "bacso" — 2006/6/22 — 18:13 — page } 172 \text { — \#6 }
$$

The projection of our quadric on $\left[K_{1}, K_{2}\right]$ is just the hyperboloid given by (3). Indeed the relation

$$
x^{2}+y^{2}-t^{2}=1-z^{2}
$$

implies that the coordinates of the points with nonzero $z$-coordinate of the quadric satisfy the inequality

$$
x^{2}+y^{2}-t^{2}<1
$$

Thus in order to represent our quadric, first we have to represent a hyperboloid of one sheet in the Monge system given by $K_{1}$ and $K_{3}$. The first projections of the points of this hyperboloid are the exterior points of a circle of radius 1 , while their third projections constitute the exterior of a hyperbola. Our claim can be seen immediately. In case of $t=0$ we obtain the circle of the equation $x^{2}+y^{2}=1$ in the image plane. If $t \neq 0$, then $x^{2}+y^{2}=1+t^{2}>1$, so the first contour is indeed a circle. The intersection of our hyperboloid with the 2-plane of equation $y=0$ is the hyperbola of equation $x^{2}-t^{2}=1$. The points with nonzero $y$-coordinate of the hyperboloid satisfy the inequality $x^{2}-t^{2}=1-y^{2}<1$, therefore the third contour is a hyperbola, as we claimed.

It can be shown analogously that the intersection of the quadric (1) with the hyperplane $\left[K_{2}, K_{3}\right]$ of equation $y=0$ is also a hyperboloid of one sheet. Thus the second projection of the quadric is also a circle with the same radius as the first projection.

To get a more expressive visualization it is worth to translate the centre of the quadric from the origin, then the individual images become more separate from each other. Thus we get two circles of the same radius whose centers are located on the same ordering line, and a hyperbola.

Now we describe how to construct the projections of a point of this quadric.

Suppose first that $P^{\prime}$ is an exterior point of the first contour circle $k_{1}^{\prime}$. We determine the second and the third projections of those points which are situated on the quadric and whose first projection is the point $P^{\prime}$. The projection on the hyperplane $\left[K_{1}, K_{3}\right]$ is a hyperboloid of one sheet. This hyperboloid may be represented in the Monge system given by $K_{1}$ and $K_{3}$, thus we obtain just the first and the second projection of our quadric. Thus in the well-known manner, we can construct points $P_{1}^{\prime \prime \prime}$ and $P_{2}^{\prime \prime \prime}$ such that $\left(P^{\prime}, P_{1}^{\prime \prime \prime}\right)$ and $\left(P^{\prime}, P_{2}^{\prime \prime \prime}\right)$ are the points of the hyperboloid of one sheet obtained by the projection on the hyperplane $\left[K_{1}, K_{3}\right]$. In the construction we use the fact that the hyperboloid in question is a hyperboloid of revolution. Thus it is enough to construct those points of its generator lines which are situated in the first trace curve and on the circle $k$, and 
to determine the points of the parallel circle which has the same radius as $k$. Due to this special location, the projection of the parallel circle in question is also $k$. We also utilize that the first projections of the generators are tangent to the least parallel circle $\left(k_{1}^{\prime}\right)$ of the hyperboloid of revolution. Thus the generators passing through a wanted point $P$ can be obtained on the first projection as tangent lines from $P^{\prime}$ to $k_{1}^{\prime}$. Their second projections can be constructed with the help of parallel circles. Finally, using an ordering line, we can intersect the wanted point $P_{1}^{\prime \prime \prime}, P_{2}^{\prime \prime \prime}$ from the generators.

To determine the second projection, we use the fact that the projection of our quadric on the hyperplane $\left[K_{2}, K_{3}\right]$ is a hyperboloid of one sheet congruent to the previous one. Thus, for example, the possible second projection of $P_{1}^{\prime \prime \prime}$ has to be situated on a parallel circle with the same radius as in the previous construction. In this way we can also obtain the wanted points $P_{1}^{\prime \prime}$ and $P_{2}^{\prime \prime}$ on the ordering line used before.

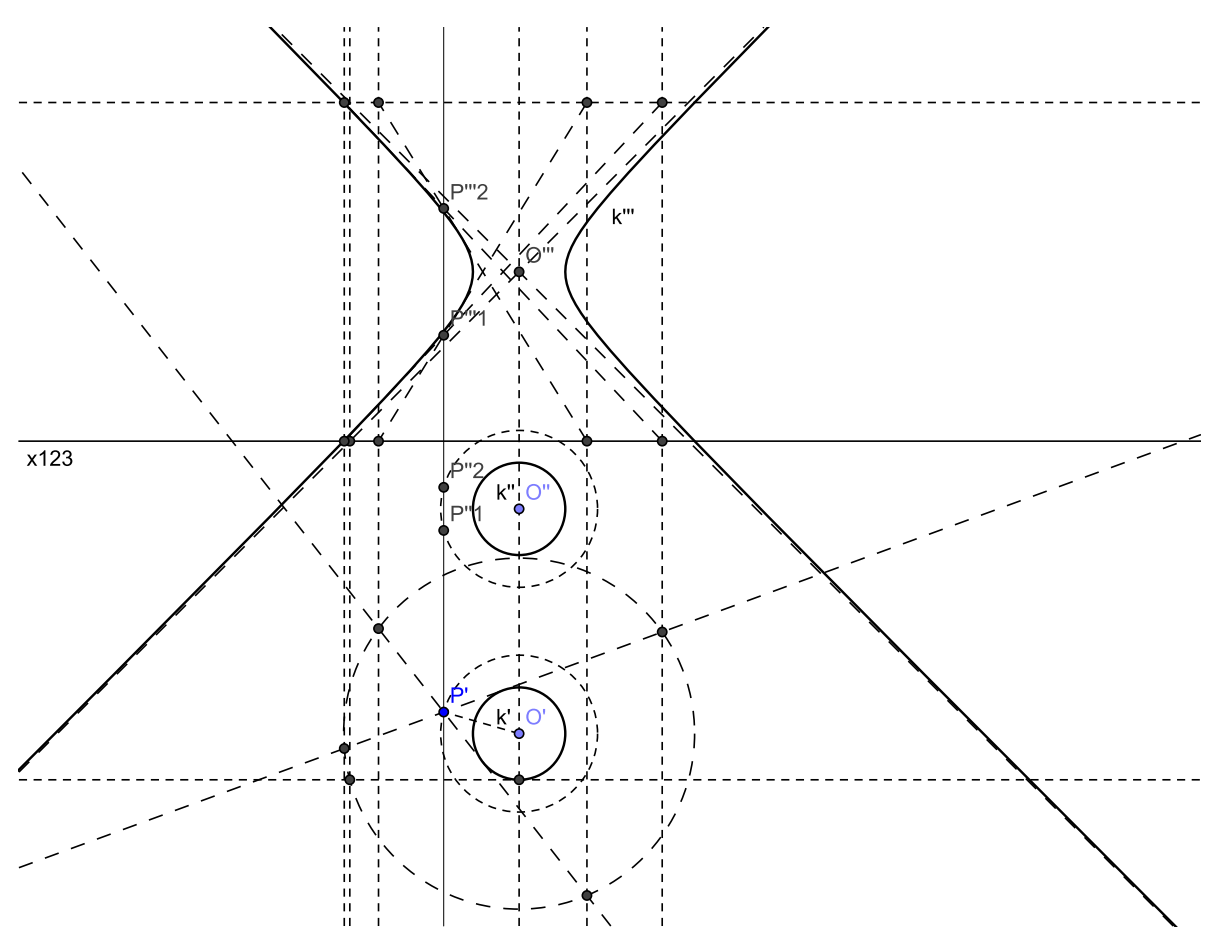

Figure 2 


$$
\text { "bacso" — 2006/6/22 — 18:13 — page } 174 \text { — \#8 }
$$

To sum up, it can be stated that, as in the case of hyperspheres, there exist four points on the quadric whose first projection is $P^{\prime}$, namely $\left(P^{\prime}, P_{1}^{\prime \prime}, P_{1}^{\prime \prime \prime}\right)$, $\left(P^{\prime}, P_{1}^{\prime \prime}, P_{2}^{\prime \prime \prime}\right),\left(P^{\prime}, P_{2}^{\prime \prime}, P_{1}^{\prime \prime \prime}\right),\left(P^{\prime}, P_{2}^{\prime \prime}, P_{2}^{\prime \prime \prime}\right)$.

4. The image of the quadric of equation $x_{1}^{2}+x_{2}^{2}+x_{3}^{2}-x_{4}^{2}-x_{5}^{2}=0$ under an axonometry

As in the traditional three-dimensional descriptive geometry, the axonometric method is also a well suited tool to visualize an object of 4-space. An axonometry is well defined by the image of an orthonormal basis of the 4-space onto a drawing plane. The image of a point under this kind of axonometry can be constructed in the same way as under an axonometric mapping of the 3-space. According to the fundamental theorem of axonometry, any axonometric mapping is the product of a parallel projection onto the drawing plane and an affine transformation of the drawing plane. A very expressive visualization of a hypersphere of the 4-space via axonometry was presented by J. Szabó in his paper [9]. An excellent analytical treatment of axonometric mappings of higher dimensional Euclidean spaces, as well as the contour of their hyperspheres can be found in [10].

In this paragraph first we are going to represent the intersections of the quadric of equation

$$
x^{2}+y^{2}+z^{2}-t^{2}=1
$$

with the projecting planes. It can be seen at once that the intersections with the first and the second projecting planes are unit circles, while the intersection with the third projecting plane is the hyperbola of equation

$$
x^{2}-t^{2}=1 \quad(y=z=0)
$$

We represent the hyperplane of equation $z=0$ in Cavalier axonometry, i.e. we use equally distorted and orthogonal axes. The hyperbola given by (4) is situated in this hyperplane, and, due to the special location, it can be seen in true length.

The intersection of the quadric with the coordinate plane $[x, y]$ is a circle. Its image under the axonometry is an ellipse, determined by the unit segments of the axes $x$ and $y$ as conjugate half-diameters.

Analogously, we also represent the hyperplane of equation $y=0$ in Cavalier axonometry. In this hyperplane the intersection of the quadric with the coordinate plane $[x, z]$ is again a circle, whose image under the axonometry is the ellipse with half-diameters as the unit segments of the axes $x$ and $z$. 


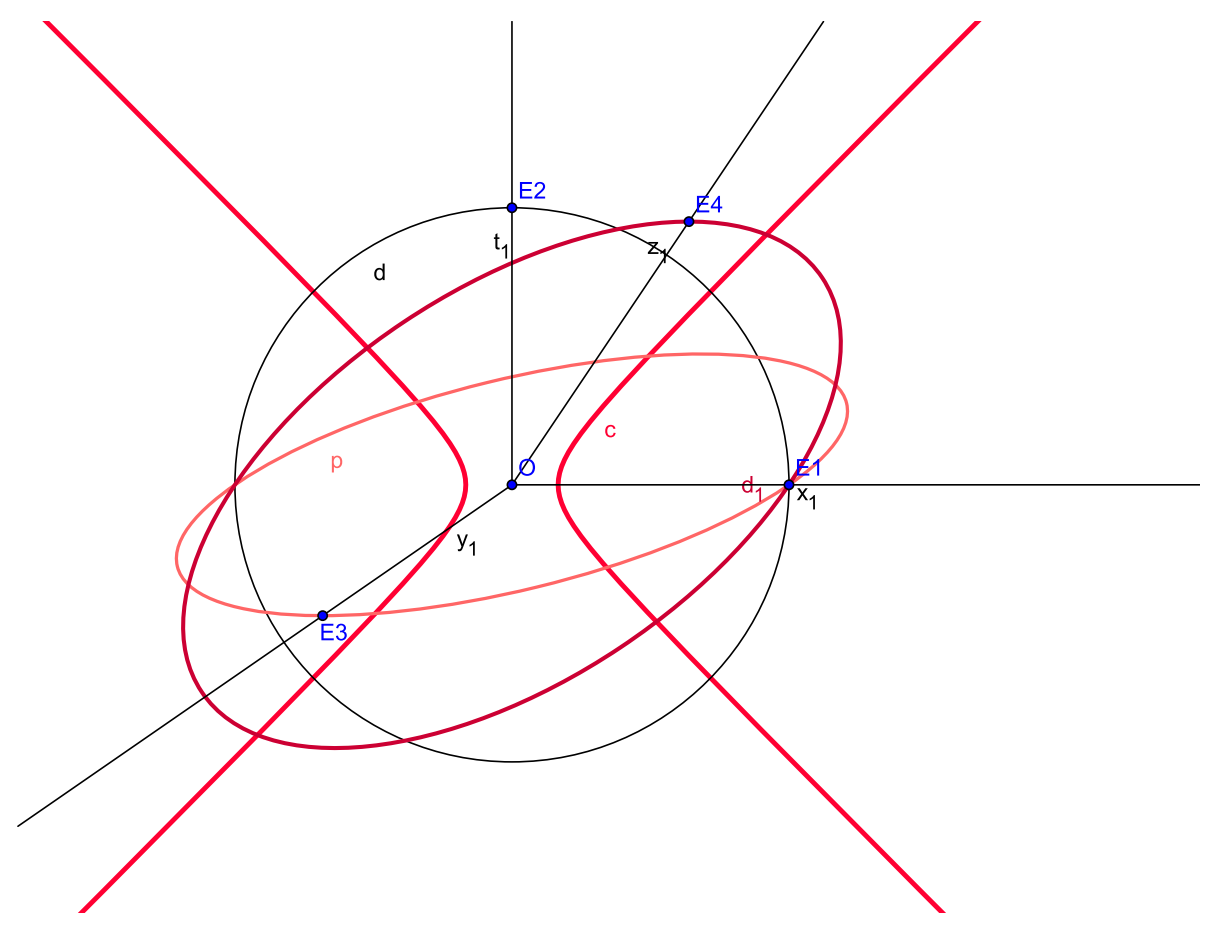

Figure 3

Finally we construct the axonometric image of the quadric. It can easily be seen that the map $f$ given by

$$
\begin{array}{r}
f(u, v, w):=\left(\sqrt{1+u^{2}} \cdot \cos v \cdot \sin w, \sqrt{1+u^{2}} \cdot \cos v \cdot \cos w, \sqrt{1+u^{2}} \cdot \sin v, u\right), \\
u \in \mathbb{R}, \quad v, w \in[0,2 \pi]
\end{array}
$$

parametrizes the quadric. Holding $u$ constant, the parameter surface is a sphere of radius $\sqrt{1+u^{2}}$ in the 3 -space. It is also clear that our quadric is a hypersurface of revolution in the 4-space, obtained by rotating a hyperboloid of one sheet of the 3 space. Thus the parallel circles used for representing points can be endowed with a four-dimensional meaning: these are the projections of the 'parallel spheres' of the hypersurface.

A possible axonometric image of the quadric can be constructed via the representation of the parameter surfaces $u=$ const., i.e., representing the spheres of center $(0,0,0, u)$, radius $\sqrt{1+u^{2}}$. We use Cavalier axonometry determined by the frame $((1,0,0,0),(0,1,0,0),(0,0,1,0))$. The image of the unit sphere is just 
the image of the parameter surface $u=0$. The axonometric image of a parameter surface $u=t_{0}$ can be obtained as follows. First, we translate the previous frame to the point $\left(0,0,0, t_{0}\right)$. Next, we construct the axonometric image of the sphere of radius $\sqrt{1+t_{0}^{2}}$ in the Cavalier-axonometry determined by the new frame.

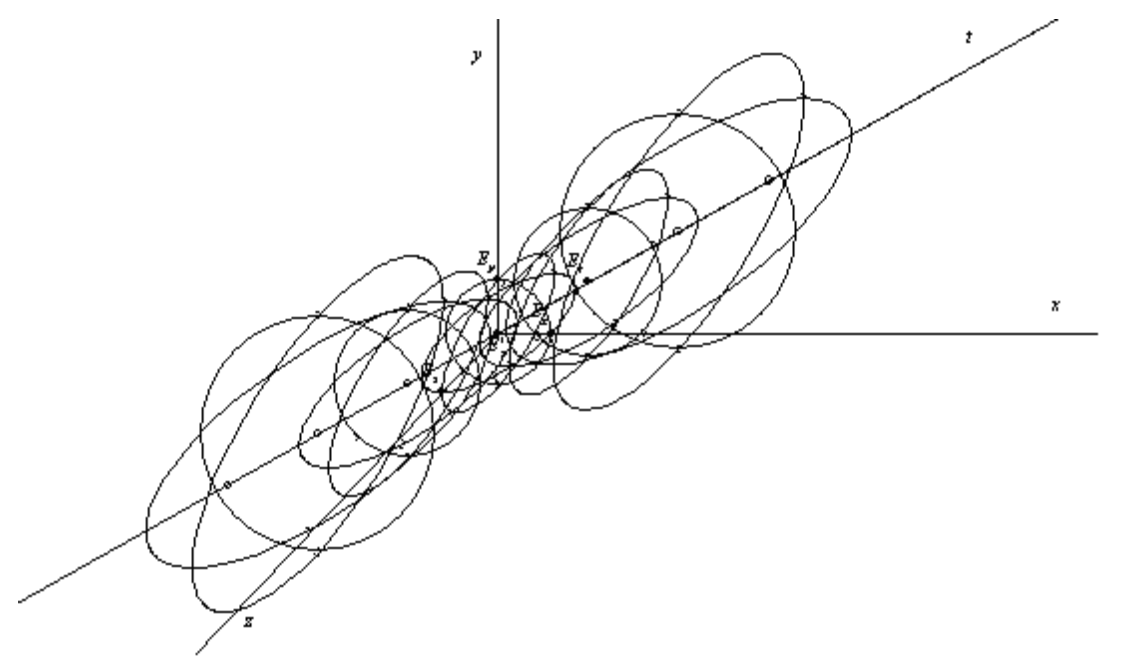

Figure 4

The hyperspheres can be represented with the help of the images of their great circles; these are ellipses whose conjugate half-diameters are the unit segments.

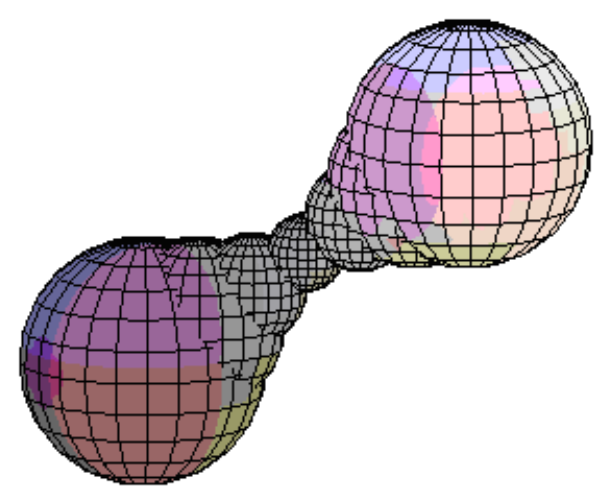

Figure 5 


$$
\text { "bacso" — 2006/6/22 — 18:13 — page } 177 \text { — \#11 }
$$

Using computer, and plotting the spheres obtained as the parameter surfaces of the quadric, we get a more decorative picture (Figure 5), but it reflects the four-dimensional character of the quadric less suggestively.

\section{Acknowledgement}

The authors are indebted to the referee for his valuable suggestions.

\section{References}

[1] Oswald Giering, Vorlesungen über höhere Geometrie, Vieweg, Braunschweig, 1982.

[2] László Gyarmathi, Die anwendung der projetierenden raumelemente bei der Maurinschen abbildung der vierdimensionalen linearen raumes, Matematikai Lapok $\mathbf{5}$, Budapest (1954), 145-150.

[3] Béla Kerékjártó, Les fondements de la géométrie. Tome 2. Géométrie projective, Gauthier-Villars-Akadémiai Kiadó, Paris-Budapest, 1966.

[4] J. Maurin, Geometrie Descriptive A Quatre Dimensions, Paris, 1948.

[5] H. P. Manning, Geometry of Four Dimensions, Dover Publ., New York, 1956.

[6] J. Tschupik, Über lineare Zweibildersysteme im projektiven $\mathbb{R}^{n}$, Monatshefte Math. 63 (1959), 214-227.

[7] J. Tschupik, Zur Theorie der konstruktiv brauchbaren linearen Bildersysteme im $\mathbb{R}^{n}$, Österreic. Akad. 170 (1962), 253-276.

[8] J. Tschupik, Über lineare Bildersysteme im projektiven $\mathbb{R}^{n}$, Monatshefte Math. 69 (1965), 339-352.

[9] József Szabó, On the axonometrical projections in the computer graphics, Acta Mathematica Academie Paedagogicae Nyíregyháziensis 17 (2001), 179-183.

[10] Günter Weiss, $(n, 2)$-Axonometries and the Contour of Hyperspheres, Journal for Geometry and Graphics 1 (1997), 157-167.

SÁNDOR BÁCSÓ and ZOLTÁN SZILASI

FACULTY OF INFORMATICS

UNIVERSITY OF DEBRECEN

H-4010 DEBRECEN

P. O. BOX 12

HUNGARY

E-mail: bacsos@inf.unideb.hu

E-mail: szilasi@math.klte.hu

(Received December, 2005) 